\title{
Detection of X-rays from the jet-driving symbiotic star Hen 3-1341 (Research Note)
}

\author{
M. Stute ${ }^{1}$, G. J. M. Luna ${ }^{2,3}$, I. F. Pillitteri ${ }^{3}$, and J. L. Sokoloski ${ }^{4}$ \\ ${ }^{1}$ Institute for Astronomy and Astrophysics, Section Computational Physics, Eberhard Karls Universität Tübingen, \\ Auf der Morgenstelle 10, 72076 Tübingen, Germany \\ e-mail: matthias.stute@tat.physik.uni-tuebingen.de \\ 2 Instituto de Astronomía y Física del Espacio (CONICET-UBA), Casilla de Correo 67, Suc. 28 (C1428ZAA), \\ Ciudad Autónoma de Buenos Aires, Argentina \\ 3 Harvard-Smithsonian Center for Astrophysics, 60 Garden St., MS 15, Cambridge, MA 02138, USA \\ ${ }^{4}$ Columbia Astrophysics Laboratory, 550 W. 220th Street, 1027 Pupin Hall, Columbia University, New York, NY 10027, USA
}

Received 9 February 2012 / Accepted 9 May 2013

\section{ABSTRACT}

\begin{abstract}
Context. Hen 3-1341 is a symbiotic binary system consisting of a white dwarf and a red giant star that is one of about ten symbiotics that show hints of jets. The bipolar jets have been detected through displaced components of emission lines during its outburst from 1998 to 2004. These components disappeared when Hen 3-1341 reached quiescence. On February 23, 2012, Hen 3-1341 started a new outburst with the emergence of new bipolar jets on March 3, 2012.

Aims. We observed Hen 3-1341 during quiescence with XMM-Newton in March 2010 with an effective exposure time of 46.8 ks and with Swift on March 8-11, 2012 as ToO observations with an effective exposure time of $10 \mathrm{ks}$ in order to probe the interaction of the jet with the ambient medium and also the accretion onto the white dwarf.

Methods. We fitted the XMM-Newton X-ray spectra with XSPEC and examined the X-ray and UV light curves.

Results. We report the detection of X-ray emission during quiescence from Hen 3-1341 with XMM-Newton. The spectrum can be fitted with an absorbed one-temperature plasma or an absorbed blackbody. We did not detect Hen 3-1341 during our short Swift exposure. Neither periodic or aperiodic X-ray nor UV variability were found.

Conclusions. Our XMM-Newton data suggest that interaction of the residual jet with the interstellar medium might survive for a long time after outbursts and might be responsible for the observed X-ray emission during quiescence. Additional data are strongly needed to confirm these suggestions.
\end{abstract}

Key words. binaries: symbiotic - white dwarfs - X-rays: stars - ISM: jets and outflows - stars: individual: Hen 3-1341 (V2523 Oph)

\section{Introduction}

Hen 3-1341 (=V2523 Oph = SS73 75) was discovered by Henize (1976), and its classification as a symbiotic star followed the spectroscopic observations by Allen (1978). The highexcitation conditions found by Allen were later confirmed by Gutierrez-Moreno et al. (1997), whose optical and ultraviolet spectra show strong emissions in the Balmer continuum, by the $\mathrm{N} \mathrm{V},[\mathrm{Fe}$ VII], He II lines, and the symbiotic band at $6830 \AA$ due to Raman scattering by neutral hydrogen. Mürset \& Schmid (1999) derived a spectral type of M4 for the cool giant. Its optical colors are typical of symbiotic stars harboring a very hot and luminous white dwarf and infrared colors appropriate to a cool giant without circumstellar dust. In quiescence, Hen 3-1341 resembles Z And, the prototype of symbiotic stars (Kenyon 1986).

About 200 symbiotic stars are known (e.g. Belczynski et al. 2000), but jets have been detected at different wavelengths only in about ten of them (Brocksopp et al. 2004). While Hen 3-1341 was in outburst, Tomov et al. (2000) discovered a jet with a radial velocity of $820 \mathrm{~km} \mathrm{~s}^{-1}$ in high-resolution spectra leading to emission components displaced on both sides of the main emission lines. Munari et al. (2005) followed the evolution of the jet emission components and reports their disappearance when the system returned to quiescence. On February 23, 2012, Hen 3-1341 started a new outburst with the emergence of new bipolar jets on March 3, 2012 (Munari et al. 2012a,b).

X-ray observations are an excellent probe of the bow and internal shocks of the jet that emit soft X-rays (photon energies $\$ 2 \mathrm{keV}$ ) and also the central parts of the jet engine, where gas is being accreted to power the jet, leading to hard (photon energies $\gtrsim 2 \mathrm{keV}$ ) and/or soft X-ray emission. Up to now, only in R Aqr (Kellogg et al. 2001, 2007) and CH Cyg (Galloway \& Sokoloski 2004; Karovska et al. 2007, 2010) have jets from symbiotic stars been resolved in X-rays. All known jet sources, when observed in X-rays, show soft components with $k T \lesssim 2 \mathrm{keV}$ : R Aqr (Kellogg et al. 2001, 2007), CH Cyg (Galloway \& Sokoloski 2004; Karovska et al. 2007, 2010), MWC 560 (Stute \& Sahai 2009), V1329 Cyg (Stute et al. 2011), RS Oph (Luna et al. 2009), AG Dra (Gonzalez-Riestra et al. 2008) and Z And (Sokoloski et al. 2006). Furthermore, the three objects CH Cyg, R Aqr, and MWC 560 also emit hard X-rays (Mukai et al. 2007; Nichols et al. 2007; Stute \& Sahai 2009). Z And showed hard emission in only one of three observations (Sokoloski et al. 2006).

The paper is organized as follows. In Sect. 2, we show details of the observations and the analysis of the data. After that we 


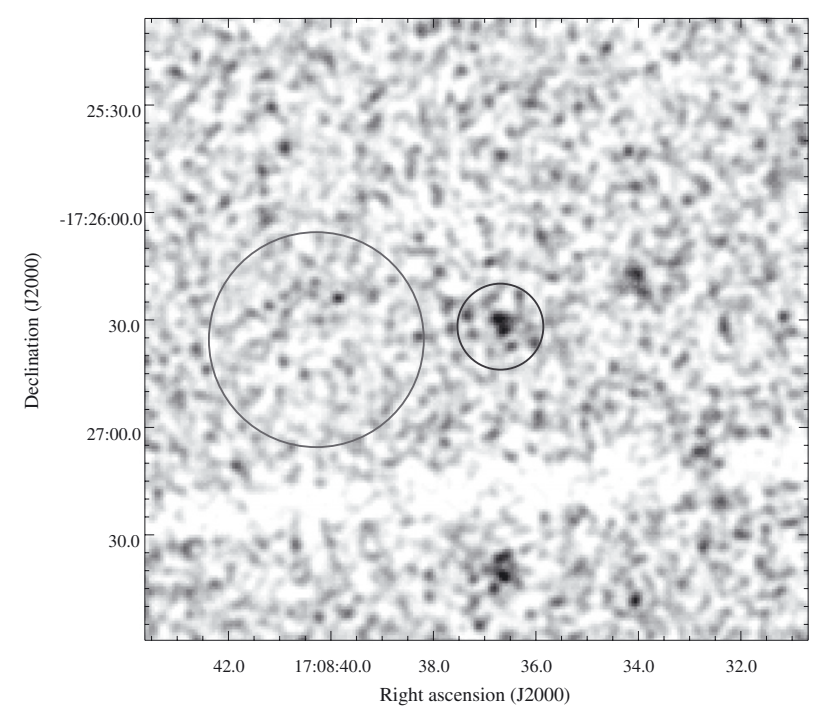

Fig. 1. XMM-Newton EPIC MOS1 + MOS2 + pn image in $0.3-8.0 \mathrm{keV}$ centered on Hen 3-1341. Extraction regions for the source and background events are overplotted.

describe the results in Sect. 3. We end with a discussion and conclusions in Sect. 4.

\section{Observation and analysis}

\subsection{XMM-Newton observations during quiescence}

We observed the field of Hen 3-1341 for $\sim 58 \mathrm{ks}$ with XMM-Newton in March 2010 using the EPIC instrument operating in full window mode and with the Medium thickness filter. Simultaneously, we used the Optical Monitor (OM). All the data reduction was performed using the Science Analysis Software (SAS) software package ${ }^{1}$ version 10.0 . We removed events collected during high background intervals. We further filtered events to keep only those with $F L A G=0$ and PATTERN in 0-4 (only single and double events) for pn and 0-12 for MOS, as prescribed in the SAS guide. The resulting exposure time after these steps is $47 \mathrm{ks}$ with pn and $58 \mathrm{ks}$ with MOS.

After first inspection of the data, only a weak source was present at the position of Hen 3-1341, therefore we used a source detection algorithm based on a multiscale wavelet convolution (Damiani et al. 1997a,b) specifically tailored for EPIC $X M M-N e w t o n$ cameras. In particular it allows the detection using the weighted sum of the images obtained with pn and MOS CCDs. We set the threshold for source detection at $4.5 \sigma$ of local background to retain, on a statistical basis, at most one spurious source per field.

The source spectra and light curves were accumulated from a circular region of $12^{\prime \prime}$ radius (240 pixels, Fig. 1) centered on Hen 3-1341 using the coordinates from SIMBAD. We extracted the background spectra and light curves from a source-free circular region with a radius of $30^{\prime \prime}$ (600 pixels) on the same chip, at the same distance from the readout node as the region from which we extracted the source counts. Spectral redistribution matrices and ancillary response files were generated using the SAS scripts rmfgen and arfgen, and spectra were fed into the spectral fitting package XSPEC ${ }^{2}$ v12.6.0. Due to the low number of counts, we did not group the counts and analyzed the

\footnotetext{
1 http://xmm.vilspa.esa.es/

2 http://heasarc.gsfc.nasa.gov/docs/xanadu/xspec/
}

ungrouped spectrum using the Cash fit statistics. For timing analysis, photon arrival times were converted to the solar system barycenter using the SAS task barycen.

\subsection{Swift observations during outburst}

We observed the field of Hen 3-1341 for $\sim 10 \mathrm{ks}$ with Swift in March 2012 as a triggered ToO observation after the reported emergence of new bipolar jets about two weeks earlier. The XRT observed in the PC (photon counting) mode. To search for X-ray emission from Hen 3-1341, we first concatenated the event files from three visits using the evselect tool, built and combined individual exposure maps using the xrtexpomap and ximage tools. Finally, we built a concatenated image and then used the ximage tool to search for source emission.

We also obtained Swift/UVOT images from which we extracted fluxes in the UVM2, UVW2, and UVW1 filters using a $5^{\prime \prime}$ source extraction region, while background flux was extracted from an annulus of the $10^{\prime \prime}$ and $20^{\prime \prime}$ inner and outer radii, respectively, around the source.

\section{Results}

\subsection{XMM-Newton}

\subsubsection{Images}

$X M M-N e w t o n$ detected the source with a significance of $6.2 \sigma$ in all EPIC images (Fig. 1). With the pn camera, we detected 564 counts in the background region, so we expect about 90 background counts within the 135 counts in the source region. Therefore the source has been detected with $4.7 \sigma$ above the background. In the MOS 1 camera, we find 141 background and 38 source counts, i.e. a detection with $3.2 \sigma$. In the MOS 2 camera, we find 125 background and 32 source counts, i.e. a detection with $2.66 \sigma$. The detected position agrees perfectly with the SIMBAD position of Hen 3-1341 and that of the closest source in the 2MASS All Sky catalog of point sources (Skrutskie et al. 2006). Other sources are considerably shifted with respect to our detected source. Thus it is very likely that the detected X-rays are indeed emitted by Hen 3-1341. The average magnitudes in the optical filters U, UVW1, and UVM2 are 12.8, 12.9 , and 13.9, respectively.

\subsubsection{Spectra}

Because of the low number of counts, we grouped the counts only for plotting purposes and not for fitting the spectrum. Furthermore, we used the Cash fit statistic as implemented in XSPEC, which allows for the analysis of background subtracted Poisson-distributed data, as discussed in Appendix B of the XSPEC manual.

The X-ray spectra of Hen 3-1341 can be described with an absorbed single-temperature plasma (wabs (apec) in XSPEC). We find $N_{\mathrm{H}} \gtrsim 8 \times 10^{21} \mathrm{~cm}^{-2}$ and $k T=0.32_{-0.12}^{+0.33} \mathrm{keV}$ (Fig. 2). The total absorbed flux between $0.3-10 \mathrm{keV}$ is $1.66 \times$ $10^{-15} \mathrm{erg} \mathrm{cm}^{-2} \mathrm{~s}^{-1}$.

We also fitted the spectrum with an absorbed blackbody model (wabs(bbody)). The fit also describes the spectra well (reduced $\chi^{2}=0.482,5$ d.o.f.), and we find $N_{\mathrm{H}}=$ $\left(5.04_{-5.11}^{+89.8}\right) \times 10^{20} \mathrm{~cm}^{-2}$ and $k T=0.24_{-0.19}^{+0.19} \mathrm{keV}$. This corresponds to a temperature of $2.8 \mathrm{MK}$ with an error range of $T=(0.6-5) \mathrm{MK}$, which is better constrained than in the 


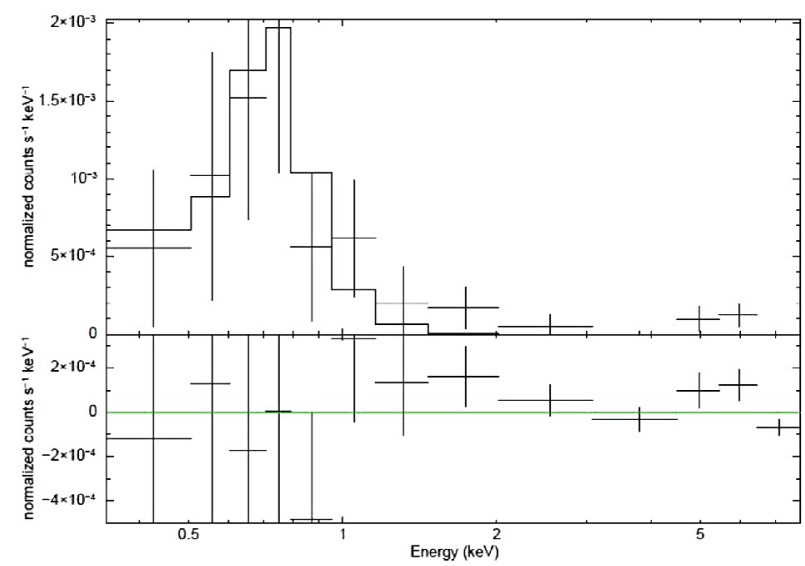

Fig. 2. Observed pn spectrum of Hen 3-1341, together with a model consisting of an absorbed one-temperature plasma (wabs (apec)).

first model. The total absorbed flux between $0.15-10 \mathrm{keV}$ is $(1.84 \pm 0.03) \times 10^{-15} \mathrm{ergs} \mathrm{cm}^{-2} \mathrm{~s}^{-1}$.

The slope of the ultraviolet spectral energy distribution clearly shows that the optical and ultraviolet fluxes are more consistent with nebular emission than blackbody emission from a hot white dwarf.

\subsubsection{Light curves}

To study the X-ray variability, we used the KolmogorovSmirnov (KS, e.g. Press et al. 1993) and Kuiper tests (Kuiper 1960) and compared the cumulative distributions of a constant model, source, and background events. The KS and Kuiper test did not detect variability (at $3 \sigma$ level) in the event arrival rate from any of the EPIC cameras.

We also examined the OM photometry with exposure times of $1500 \mathrm{~s}$ and $3000 \mathrm{~s}$. The measured rms variations in the count rates are consistent with those expected from Poisson statistics.

\subsection{Swift}

We did not detect Hen 3-1341 with our short-exposure Swift observation. The $3 \sigma$ upper limit is $1.3 \times 10^{-3}$ counts/s. Again using the two best-fit models mentioned above, this count rate would correspond to an absorbed flux of about $2.8 \times 10^{-14} \mathrm{erg} \mathrm{cm}^{-2} \mathrm{~s}^{-1}$ and an unabsorbed flux of about $5 \times 10^{-14} \mathrm{erg} \mathrm{cm}^{-2} \mathrm{~s}^{-1}$.

With UVOT, we find average UV count rates of $74 \pm$ 8 counts/s (UVM2, $2246 \AA$ ), $224 \pm 9$ counts/s (UVW1, $2600 \AA$ ), and $141 \pm 8$ (UVW2, $1928 \AA$ ). Converted to magnitudes, these values correspond to $12.14 \pm 0.03,11.56 \pm 0.03$, and $12.01 \pm 0.03$, respectively.

\section{Discussion and conclusions}

Hen 3-1341 shows a soft X-ray emission. One possible explanation can be given in light of simulations of jets in symbiotic stars (Stute 2006; Stute \& Sahai 2007) or protostellar jets (e.g. Bonito et al. 2010, 2011) where soft X-ray emission arises from internal shocks and the bow shock. The velocity of the shock, $v_{\text {shock}}$, can be derived using the measured temperature of the component and the following relation (assuming strong shock conditions):

$T_{\text {post shock }}=\frac{3}{16} \frac{\mu m_{\mathrm{P}}}{k_{\mathrm{B}}} v_{\text {shock }}^{2}=0.105 \mathrm{keV}\left(\frac{v_{\text {shock }}}{300 \mathrm{~km} \mathrm{~s}^{-1}}\right)^{2}$ with $m_{\mathrm{p}}$ the proton mass, $k_{\mathrm{B}}$ the Boltzmann constant, and $\mu=0.6$ the mean particle weight. Therefore the observed soft component temperature range using the APEC model corresponds to a shock velocity range of $400-750 \mathrm{~km} \mathrm{~s}^{-1}$. Tomov et al. (2000) discovered a jet with a radial velocity of $820 \mathrm{~km} \mathrm{~s}^{-1}$ while Hen 3-1341 was in outburst. The displaced spectral component then gradually disappeared when the system returned to quiescence in 2004 (Munari et al. 2005). Since then the outflow has likely been decelerated by the interstellar medium. Thus it is possible that the soft component is emitted by residuals of this interaction.

Although we know that a jet had been present in this object, other scenarios for explaining the emission of soft X-rays are possible:

- One scenario is photospheric emission from a hot white dwarf. The observed UV flux could in principle be produced by a white dwarf with a radius of $0.14 R_{\odot}$ and a luminosity of $3000 L_{\odot}$, as proposed by Munari et al. (2005), but the X-ray emission cannot. An effective temperature of $2.8 \mathrm{MK}$, which would be required to produce the observed $\mathrm{X}$-ray emission, is inconsistent with the optical emission-line spectrum (Munari et al. 2005).

- Another possible source of the detected X-rays may be colliding winds (Mürset et al. 1997), since soft X-ray emission has also been detected in objects without jets (Mürset et al. 1997). We disfavor this picture because no wind signatures have been detected in spectroscopic observations during quiescence or outburst (van Winckel et al. 1993; Tomov et al. 2000) - only signatures of a bipolar jet during the outbursts in 1999 and 2012 (Tomov et al. 2000; Munari et al. 2012b).

We performed hydrodynamical simulations of the propagating jet with parameters representative of Hen 3-1341 (Stute et al., in prep.), in which we varied the jet mass-loss rate following the observed AAVSO light curve. Temperature and density maps from our simulations are used, together with emissivities calculated with the atomic database ATOMDB, for estimating the $\mathrm{X}$-ray luminosity emitted by the jet. Although we made simple assumptions, we can reproduce the order of magnitude of the observed X-ray luminosity.

The X-ray and the optical light curves are consistent with a constant flux. The measured rms variations in the count rates are consistent with those expected from Poisson statistics.

Using the effective exposure time of $46.8 \mathrm{ks}$ in all instruments, the sensitivity of XMM-Newton (Watson et al. 2001) gives an upper limit of about $10^{-14} \mathrm{erg} \mathrm{s}^{-1} \mathrm{~cm}^{-2}$ in the hard band ( $\gtrsim 2 \mathrm{keV}$ ). Using a distance of $3.1 \mathrm{kpc}$ (Gutierrez-Moreno et al. 1997), the flux upper limit gives an upper limit for the the hard $\mathrm{X}$-ray luminosity of about $10^{31} \mathrm{erg} \mathrm{s}^{-1}$. Munari et al. (2005) estimats a white dwarf mass of $0.4 M_{\odot}$, a radius of $0.1 R_{\odot}$ and an accretion rate of $5 \times 10^{-8} M_{\odot} \mathrm{yr}^{-1}$, which would lead to

$L_{\mathrm{x}} \lesssim \frac{G M_{\mathrm{WD}} \dot{M}}{2 R_{\mathrm{WD}}}=1.2 \times 10^{34} \mathrm{erg} \mathrm{s}^{-1}$.

Therefore either the accretion rate has decreased substantially or the accretion luminosity from the boundary layer is emitted in other wavelength bands besides the X-rays; i.e., the accretiondisk boundary layer is optically thick, as in Mira - another jetproducing symbiotic star with very low X-ray flux (Sokoloski \& Bildsten 2010). In any case, the accretion rate estimate agrees with Hen 3-1341 being in quiescence.

Unfortunately, we did not detect Hen 3-1341 with our shortexposure Swift observation. The derived upper limit shows that 
the soft X-ray component has not increased its flux by a factor higher than about 20. This neither supports nor rejects our conclusions. The Swift observations took place about one week after the reported emergence of the jets. In our simulations we found that it takes the X-ray luminosity up to 20 days to increase significantly after a new emerged jet has started interacting with the surrounding medium (Stute \& Sahai 2007; Stute et al., in prep.). Furthermore, the magnitude of the increase depends on the jet parameters.

We have to note that uncertainties in the distance might change the flux levels; however, Gutierrez-Moreno et al. (1997) estimate an error of their derived distance of only $4 \%$.

New data are strongly needed to confirm our conclusion.

Acknowledgements. We acknowledge helpful comments and suggestions by an anonymous referee. This work is based on observations obtained with XMM-Newton, an ESA science mission with instruments and contributions directly funded by ESA Member States and the USA (NASA). We thank NASA for funding this work through XMM-Newton AO-8 awards NNX09AP88G to GJML and JLS, and NNX10AK31G to J.L.S.. We gratefully acknowledge the effort of the entire Swift team for the execution of our ToO request. This publication makes use of data products from the Two Micron All Sky Survey, which is a joint project of the University of Massachusetts and the Infrared Processing and Analysis Center/California Institute of Technology, funded by the National Aeronautics and Space Administration and the National Science Foundation. We acknowledge with thanks the variable star observations from the AAVSO International Database contributed by observers worldwide and used in this research.

\section{References}

Allen, D. A. 1978, MNRAS, 184, 601

Belczynski, K., Mikolajewska, J., Munari, U., et al. 2000, A\&AS, 146, 407

Bonito, R., Orlando, S., Miceli, M., et al. 2010, A\&A, 517, A68

Bonito, R., Orlando, S., Miceli, M., et al. 2011, ApJ, 737, 54

Brocksopp, C., Sokoloski, J. L., Kaiser, C., et al. 2004, MNRAS, 347, 430
Damiani, F., Maggio, A., Micela, G., \& Sciortino, S. 1997a, ApJ, 483, 350 Damiani, F., Maggio, A., Micela, G., \& Sciortino, S. 1997b, ApJ, 483, 370 Galloway, D. K., \& Sokoloski, J. L. 2004, ApJ, 613, L61

Gonzalez-Riestra, R., Viotti, R. F., Iijima, T., et al. 2008, A\&A, 481, 725

Gutierrez-Moreno, A., Moreno, H., Costa, E., et al. 1997, ApJ, 485, 359

Henize, K. G. 1976, ApJS, 30, 491

Karovska, M., Carilli, C. L., Raymond, J. C., \& Mattei, J. A. 2007, ApJ, 661, 1048

Karovska, M., Gaetz, T. J., Carilli, C. L., et al. 2010, ApJ, 710, 132

Kellogg, E., Pedelty, J. A., \& Lyon, R. G. 2001, ApJ, 563, 151

Kellogg, E., Anderson, C., Korreck, K, et al. 2007, ApJ, 664, 1079

Kenyon, S. J. 1986, The symbiotic stars (Cambridge University Press)

Kuiper, N. H. 1960, in Tests concerning random points on a circle, Proc. of the Koninkl. Nederl. Akad. van Wetenschappen, Ser. A., 63, 38

Luna, G. J. M., Montez, R., Sokoloski, J. L., Mukai, K., \& Kastner, J. H. 2009, ApJ, 707, 1168

Mürset, U., \& Schmid, H. M. 1999, A\&AS, 137, 473

Mürset, U., Wolff, B., \& Jordan, S. 1997, A\&A, 319, 201

Mukai, K., Ishida, M., Kilbourne, C., et al. 2006, PASJ, 59, 177

Munari, U., Siviero, A., \& Henden, A. 2005, MNRAS, 360, 1257

Munari, U., Milani, A., Siviero, A., et al. 2012a, ATEL, \#3946

Munari, U., Valisa, P., Dallporta, S., et al. 2012b, ATEL, \#3952

Nichols, J. S., DePasquale, J., Kellogg, E., et al. 2007, ApJ, 660, 651

Popham, R., \& Narayan, R. 1995, ApJ, 442, 337

Press, W. H., Teukolsky, S. A., Vetterling, W. T., \& Flannery, B. P. 1993, Numerical Recipes in C: The Art of Scientific Computing, 2nd edn. (Cambridge: Cambridge University Press)

Skopal, A., Sekeras, M., Gonzalez-Riestra, R., \& Viotti, R. F. 2009, A\&A, 507, 1531

Skopal, A., Tarasova, T. N., Carikova, Z., et al. 2011, A\&A, 536, 27

Skrutskie, M. F., Cutri, R. M., Stiening, R., et al. 2006, ApJ, 131, 1163

Sokoloski, J. L., \& Bildsten, L. 2010, ApJ, 723, 1188

Sokoloski, J. L., Kenyon, S. J., Espey, B. R., et al. 2006, ApJ, 636, 1002

Stute, M. 2006, A\&A, 450, 645

Stute, M., \& Sahai, R. 2007, ApJ, 665, 698

Stute, M., \& Sahai, R. 2009, A\&A, 498, 209

Stute, M., Luna, G. J. M., \& Sokoloski, J. L. 2011, ApJ, 731, 12

Tomov, T., Munari, U., \& Marrese, P. M. 2000, A\&A, 354, L25

van Winckel, H., Duerbeck, H. W., \& Schwarz, H. E. 1993, A\&AS, 102, 401 